\title{
SATISFAÇÃO PROFISSIONAL: PERCEPÇÃO DE ENFERMEIROS DE UM HOSPITAL UNIVERSITÁRIO
}

\author{
Amanda Ceschini Rigue ${ }^{1}$, Graziele de Lima Dalmolin², Katiane Sefrin Speroni ${ }^{3}$, Julia Zancan Bresolin ${ }^{4}$, \\ Andreia Aldair Rigue ${ }^{5}$
}

\begin{abstract}
RESUMO: Objetivou-se investigar a satisfação profissional percebida de enfermeiros de uma instituição hospitalar do Sul do Brasil. Pesquisa quantitativa, desenvolvida com 144 enfermeiros de um hospital universitário, no período de dezembro de 2014 a fevereiro de 2015, utilizando-se o Índice de Satisfação Profissional. Os resultados evidenciaram que $79,2 \%(n=114)$ dos enfermeiros referem possuir autonomia, porém $47,9 \%(n=69)$ precisam realizar atividades que vão contra seu melhor julgamento em algumas situações. Para 75,7\% ( $n=109)$ a interação com equipe de enfermagem é satisfatória, enquanto para 70,1\% ( $n=101)$ a interação com médicos é permeada pela desvalorização do trabalho e conhecimentos. Quanto ao salário atual, $79,2 \%$ ( $n=114)$ o consideram satisfatório; $93,8 \%(n=135)$ consideram seu trabalho importante; $88,2 \%(n=127)$ estão satisfeitos com o trabalho realizado, e, 80,6\% $(n=116)$ consideram que as atividades burocráticas dificultam uma assistência mais qualificada. Ressaltamos a importância das instituições avaliarem as necessidades dos profissionais e buscarem estratégias para aumentar sua satisfação.
\end{abstract}

DESCRITORES: Assistência de enfermagem; Enfermagem; Satisfação no trabalho; Saúde do trabalhador.

\section{WORK SATISFACTION: PERCEPTION OF NURSES OF A UNIVERSITY HOSPITAL}

\begin{abstract}
The aim of this study was to investigate the perceived work satisfaction of nurses in a hospital in southern Brazil. This quantitative study was conducted with 144 nurses of a university hospital, from December 2014 to February 2015, using the Index of Work Satisfaction. The results showed that $79.2 \%(n=114)$ of the nurses reported having autonomy, however, $47.9 \%(n=69)$ need to carry out activities that went against their better judgment in some situations. For $75.7 \%(n=109)$ the interaction with the nursing staff was satisfactory, while for $70.1 \%(n=101)$ the interaction with doctors was permeated by the devaluation of their work and knowledge. Regarding the salary, $79.2 \%(n=114)$ considered it satisfactory; $93.8 \%(n=135)$ considered their work important; $88.2 \%$ ( $n$ $=127)$ were satisfied with the work performed and $80.6 \%(n=116)$ considered that bureaucratic activities hampered more qualified care. The importance is highlighted of the institutions evaluating the needs of professionals and seeking strategies to increase their satisfaction.
\end{abstract}

DESCRIPTORS: Nursing care; Nursing; Work satisfaction; Worker's health.

\section{SATISFACCIÓN PROFESIONAL: PERCEPCIÓN DE ENFERMEROS DE UN HOSPITAL UNIVERSITARIO}

RESUMEN: El objetivo del estudio fue investigar la satisfacción profesional percebida por enfermeros de una institución hospitalar del Sur de Brasil. Investigación cuantitativa, desarrollada con 144 enfermeros de un hospital universitario, en el periodo de diciembre de 2014 a febrero de 2015, utilizándose el Índice de Satisfacción Profesional. Los resultados apuntan que 79,2\% (n=114) de los enfermeros afirman presentar autonomía, pero 47,9\% (n=69) necesitan realizar actividades que no están de acuerdo a lo que piensan ser mejor en algunas situaciones. Para $75,7 \%(n=109)$, la interacción con el equipo de enfermería es satisfactoria, mientras para $70,1 \%$ $(n=101)$ la interacción con médicos muestra desvalorización del trabajo y conocimientos. Acerca del sueldo actual, $79,2 \%(n=114)$ lo creen satisfactorio; $93,8 \%(n=135)$ consideran su trabajo importante; $88,2 \%(n=127)$ están satisfechos con el trabajo realizado, y, $80,6 \%(n=116)$ piensan que las actividades burocráticas dificultan una asistencia más calificada. Se destaca la importancia de las instituciones de evaluar las necesidades de los profesionales y buscar estrategias para aumentar su satisfacción.

DESCRIPTORES: Asistencia de enfermería; Enfermería; Satisfacción en trabajo; Salud del trabajador.

${ }^{1}$ Enfermeira. Enfermeira Assistencial na Prefeitura Municipal de Restinga Sêca. Restinga Sêca, RS, Brasil.

${ }^{2}$ Enfermeira. Doutora em Enfermagem. Docente de Enfermagem da Universidade Federal de Santa Maria. Santa Maria, RS, Brasil.

${ }^{3}$ Enfermeira. Mestre em Enfermagem. Enfermeira Assistencial no Hospital Universitário de Santa Maria. Santa Maria, RS, Brasil. ${ }^{4}$ Enfermeira. Mestranda em Enfermagem. Universidade Federal de Santa Maria. Santa Maria, RS, Brasil.

${ }^{5}$ Enfermeira. Enfermeira Assistencial no Hospital de Clínicas de Porto Alegre. Porto Alegre, RS, Brasil.

Autor Correspondente:

Graziele de Lima Dalmolin

Universidade Federal de Santa Maria

Av. Roraíma 1000 - 97105-900 - Santa Maria, RS, Brasil

E-mail: grazi.dalmolin@gmail.com
Recebido: 06/04/2016

Finalizado: 19/07/2016 


\section{INTRODUÇÃO}

A crescente globalização e a evolução no processo de trabalho estabelecem que os profissionais precisem estar cada vez mais qualificados e devam executar seus papeis de maneira efetiva. No domínio da área da saúde não é diferente, pois com a progressiva evolução da tecnologia, crescimento da demanda de alta complexidade e envelhecimento populacional, faz-se necessário que os profissionais da saúde obtenham inúmeros conhecimentos e constante atualização de saberes para o aperfeiçoamento da assistência ${ }^{(1)}$.

O cenário capitalista é marcado pela competitividade e busca por um alto nível de produtividade com baixo custo na produção. Pode ser observado, em alguns casos, o aumento do ritmo do trabalho e descaso com a satisfação profissional ${ }^{(2)}$. Por outro lado, a satisfação e o bem-estar no trabalho são vistos em alguns serviços como uma necessidade, pois a satisfação profissional é ligada à produtividade e vista como um atributo para vencer os entraves da competitividade e busca pela qualidade ${ }^{(3)}$.

A relação que o profissional estabelece com o trabalho podem refletir em sua saúde, prazer, satisfação e qualidade de vida. Estes fatores dependem de como o trabalho é compreendido, os significados e importância atribuídos pelo profissional e a maneira como ele está inserido no ambiente de trabalho ${ }^{(4)}$.

A satisfação profissional vem sendo definida de diferentes maneiras ao longo dos anos, devido ao fato da satisfação no trabalho ser complexa e de difícil conceituação. Por tratar de um estado subjetivo e individual, pode ser interpretada de diferentes maneiras. Neste sentido, também pode variar em função de influências ou fatores internos e externos do ambiente laboral, como: condições do trabalho, normas da empresa, salário e relação com os colegas e chefia ${ }^{(5)}$.

No âmbito do trabalho da enfermagem, que é desenvolvido amplamente no serviço hospitalar, a satisfação profissional é de suma importância, pois os enfermeiros assumem a responsabilidade de assistir os pacientes ininterruptamente ${ }^{(2)}$. Desta forma, se os profissionais estiverem satisfeitos, terão mais motivação para concretizar seu trabalho com qualidade, otimizando a assistência prestada.

O índice de satisfação dos profissionais pode influenciar na harmonia e estabilidade psicológica do sujeito. Desta forma, a satisfação avaliada de maneira insuficiente pode trazer implicações negativas na atuação do profissional frente ao trabalho(6) ${ }^{(6)}$ relacionado à enfermagem, também pode afetar a qualidade da assistência prestada.

Nesse sentido, é importante destacar a necessidade de avaliação da satisfação profissional, por meio de instrumentos específicos. A exemplo disto cita-se o Índice de Satisfação Profissional (ISP), que é destinado ao estudo da satisfação profissional de enfermeiros e se propõe a ser estatisticamente válido, confiável, de fácil compreensão e utilizado em benefício da equipe de enfermagem ${ }^{(7)}$.

A satisfação profissional pode influenciar no desempenho dos trabalhadores, repercutir em sua saúde e relações no ambiente de trabalho. Assim, este estudo se justificou considerando conhecer as percepções sobre a realidade vivida pelos enfermeiros em seu ambiente laboral e também na identificação de variáveis que influenciam em sua satisfação profissional. Esta pesquisa busca responder ao seguinte questionamento: Qual é a percepção da satisfação profissional de enfermeiros que atuam em uma instituição hospitalar no sul do Brasil? Definiu-se o objetivo de investigar a satisfação profissional percebida de enfermeiros, de uma instituição hospitalar do Sul do Brasil, de acordo com as dimensões do ISP.

\section{○ MÉTODO}

Trata-se de um estudo de abordagem quantitativa, do tipo Survey, realizado em um Hospital Universitário do Estado do Rio Grande do Sul. A população foi constituída por 228 enfermeiros atuantes na instituição. Adotou-se como critério de inclusão: ser enfermeiro, atuante por no mínimo trinta dias na referida instituição. O critério de exclusão restringiu-se a ausência no ambiente de trabalho no período de coleta de dados, seja por afastamentos ou férias.

Neste estudo foi utilizado uma amostra não-probabilística por conveniência, porém para reduzir 
possíveis vieses diante do tamanho da amostra, adotou-se o critério de seleção do tamanho amostral ${ }^{(8)}$ que objetivou verificar o número mínimo de indivíduos que precisavam fazer parte da amostra,para possibilitar a efetivação de exames estatísticos. É representada pela seguinte expressão matemática:

$$
\frac{n=x^{2} \cdot N \cdot P(1-P)}{d^{2}(N-1)+x^{2} \cdot P(1-P)}
$$

Em que: $\mathrm{n}=$ tamanho da amostra; $\mathrm{X}^{2}=$ valor do qui-quadrado para 1 grau de liberdade ao nível de confiança de 0,05 e que é igual a 3,89 (valor fixo pré-determinado); $\mathrm{N}=0$ tamanho da população; $\mathrm{P}=$ a proporção da população que se deseja estimar (pressupõe-se que seja de 0,50 uma vez que esta proporção forneceria o tamanho máximo amostral); $d=0$ grau de precisão expresso em proporção $(0,05)$. Assim, partindo do número total de 228 enfermeiros, obteve-se como resultado uma amostra com um número mínimo de 144 participantes.

A coleta de dados, que compreendeu o período de dezembro de 2014 a fevereiro de 2015, deuse por meio da aplicação de dois questionários, um sobre dados sociodemográficos e laborais para caracterizar os participantes e, outro, denominado Índice de Satisfação Profissional (ISP), para investigar a satisfação profissional ${ }^{(7)}$. A coleta foi realizada pela autora do estudo e por acadêmicos e mestrandos de enfermagem.

Os enfermeiros foram convidados a participar do estudo, os coletores apresentaram os objetivos, forma de participação e também entregaram os Termos de Consentimento Livre e Esclarecido (TCLE). Os questionários permaneciam com os enfermeiros, para que entregassem em data pré-agendada, após eram realizadas três tentativas para recuperação dos questionários.

O ISP foi desenvolvido em 1997 em território norte americano(7) e adaptado, validado e traduzido para a língua portuguesa em $1999^{(9)}$. Trata-se de um questionário reorganizado em duas partes (A e B). O instrumento possibilita identificar a importância atribuída pelos enfermeiros com relação a seis componentes do trabalho: Autonomia, Interação, Status Profissional, Requisitos do Trabalho, Normas Organizacionais e Remuneração(10).

A análise dos dados do ISP foi realizada incluindo somente a Parte B (Escala de Atitudes), que avalia a satisfação profissional percebida por esses enfermeiros em relação ao seu trabalho ${ }^{(10)}$. Para a análise foram agrupadas as questões conforme os componentes apresentados. Foram criadas matrizes de distribuição de frequências, agrupando as respostas "concordo", "discordo" e "neutro".

Para construção do banco de dados foi utilizado o programa Epilnfo 6.4 com dupla digitação independente, e posterior correção de erros e inconsistências. Após essa verificação, para análise dos dados utilizou-se de estatística descritiva, com distribuição de frequências relativas e absolutas, empregando o software estatístico PASW Statistics (Predictive Analytics Software), da Statistical Package for Social Sciences (SPSS), versão 21.0 para Windows.

Para o desenvolvimento da pesquisa, foram observados os aspectos éticos, conforme Resolução $466 / 12^{(11)}$. O presente estudo foi analisado e aprovado pelo Comitê de Ética em Pesquisa da Universidade Federal de Santa Maria (CEP/UFSM), sob o número do CAAE 24330213.8.0000.5346 e número do parecer de aprovação 558.262.

\section{RESULTADOS}

Dos 144 enfermeiros participantes do estudo, a predominância foi do sexo feminino ( $n=130,90,3 \%)$, com faixa etária majoritariamente entre 31 a 40 anos $(n=61,42,4 \%)$. Em relação ao estado civil ( $n=97$, $67,4 \%)$ eram casados ou possuíam companheiro e ( $n=54,37,5 \%)$ não tinham filhos.

A unidade de serviço hospitalar com maior percentual de enfermeiros foi a Unidade de Terapia Intensiva (UTI), incluindo Unidade Cardio Intensiva (UCI), UTI adulta, pediátrica e neonatal ( $\mathrm{n}=23$, $16 \%$ ). A maioria dos participantes do estudo não possuía outro emprego ( $n=128,88,9 \%$ ). Quanto ao tempo de formação profissional, a predominância foi de 1 a 10 anos ( $n=55,38,2 \%)$, o tempo de serviço na instituição foi equivalente de 1 a 10 anos $(n=45,31,3 \%)$ e o tempo de trabalho na unidade atual 
também resultou de 1 a 10 anos ( $n=64,44,4 \%)$.

O Quadro 1 apresenta a distribuição das respostas do componente autonomia, de acordo com o ISP.

Quadro 1 - Distribuição das respostas do componente Autonomia. Santa Maria, RS, Brasil, 2015

\begin{tabular}{|c|c|c|c|c|c|}
\hline AUTONOMIA & & Concordo & Neutro & Discordo & Total \\
\hline \multirow{2}{*}{$\begin{array}{l}\text { 7. Eu sinto que sou supervisionada(o) mais diretamente } \\
\text { ('de perto') do que o necessário. }\end{array}$} & $\mathbf{n}$ & 22 & 21 & 101 & 144 \\
\hline & $(\%)$ & $(15,3)$ & $(14,6)$ & $(70,1)$ & $(100)$ \\
\hline \multirow{2}{*}{$\begin{array}{l}\text { 13. Eu sinto que tenho participação suficiente no } \\
\text { planejamento da assistência para cada um dos meus } \\
\text { pacientes. }\end{array}$} & $\mathbf{n}$ & 114 & 8 & 22 & 144 \\
\hline & $(\%)$ & $(79,2)$ & $(5,6)$ & $(15,3)$ & $(100)$ \\
\hline \multirow[t]{2}{*}{ 17. Eu tenho muitas responsabilidades e pouca autoridade. } & $\mathbf{n}$ & 58 & 13 & 73 & 144 \\
\hline & $(\%)$ & $(40,3)$ & (9) & $(50,7)$ & $(100)$ \\
\hline \multirow{2}{*}{$\begin{array}{l}\text { 20. Em meu serviço, minha(s) chefia(s) toma }(\mathrm{m}) \text { todas } \\
\text { as decisões. Eu tenho pouco controle direto sobre meu } \\
\text { próprio trabalho. }\end{array}$} & $\mathbf{n}$ & 33 & 11 & 100 & 144 \\
\hline & $(\%)$ & $(22,9)$ & $(7,6)$ & $(69,4)$ & $\begin{array}{l}(100) \\
\end{array}$ \\
\hline \multirow{2}{*}{$\begin{array}{l}\text { 26. Uma grande dose de independência é permitida, se } \\
\text { não requerida por mim. }\end{array}$} & $n$ & 53 & 45 & 46 & 144 \\
\hline & $(\%)$ & $(36,8)$ & $(31,3)$ & $(31,9)$ & $(100)$ \\
\hline \multirow{2}{*}{$\begin{array}{l}\text { 30. Algumas vezes sinto-me frustrada(o) porque todas } \\
\text { as minhas atividades parecem ser pré-programadas para } \\
\text { mim. }\end{array}$} & $n$ & 47 & 17 & 80 & 144 \\
\hline & $(\%)$ & $(32,6)$ & $(11,8)$ & $(55,6)$ & $(100)$ \\
\hline \multirow{2}{*}{$\begin{array}{l}\text { 31. No meu trabalho, algumas vezes, tenho que fazer coisas } \\
\text { que vão contra meu melhor julgamento profissional. }\end{array}$} & $n$ & 69 & 14 & 61 & 144 \\
\hline & $(\%)$ & $(47,9)$ & $(9,7)$ & $(42,4)$ & $(100)$ \\
\hline \multirow{2}{*}{$\begin{array}{l}\text { 43. Em meu trabalho, tenho liberdade para tomar decisões } \\
\text { importantes, que considero apropriadas, e conto com } \\
\text { minha(s) chefia(s) para me apoiar. }\end{array}$} & $n$ & 93 & 15 & 36 & 144 \\
\hline & $(\%)$ & $(64,6)$ & $(10,4)$ & (25) & (100) \\
\hline
\end{tabular}

De acordo com os resultados obtidos, os enfermeiros participantes do estudo referem possuir autonomia em suas atividades, porém algumas vezes precisam proceder em algo contrário ao seu melhor julgamento profissional. Isto se evidencia nas afirmativas: "q13: Eu sinto que tenho participação suficiente no planejamento da assistência para cada um dos meus pacientes" (79,2\% de concordância), "q31: No meu trabalho, algumas vezes, tenho que fazer coisas que vão contra meu melhor julgamento profissional" (47,9\% de concordância).

O Quadro 2 demonstra a distribuição das respostas do componente interação, de acordo com o ISP.

Quadro 2 - Distribuição das respostas do componente Interação. Santa Maria, RS, Brasil, 2015 (continua)

\section{INTERAÇÃO PROFISSIONAL}

\begin{tabular}{|l|c|c|c|c|c|}
\hline Interação com equipe médica & & Concordo & Neutro & Discordo & Total \\
\hline $\begin{array}{l}\text { 6. Em minha unidade, os médicos geralmente cooperam } \\
\text { com a equipe de enfermagem. }\end{array}$ & $\mathbf{n}$ & 97 & 10 & 37 & 144 \\
\cline { 2 - 6 } & $\mathbf{( \% )}$ & $(67,4)$ & $(6,9)$ & $(25,7)$ & $(100)$ \\
\hline $\begin{array}{l}\text { 19. Em minha unidade, há muito trabalho em equipe } \\
\text { entre Enfermeira(o)s e médicos. }\end{array}$ & $\mathbf{n}$ & 81 & 12 & 51 & 144 \\
\cline { 2 - 6 } & $\mathbf{( \% )}$ & $(56,3)$ & $(8,3)$ & $(35,4)$ & $(100)$ \\
\hline $\begin{array}{l}\text { 35. Eu gostaria que os médicos daqui mostrassem mais } \\
\text { respeito pelas habilidades e conhecimentos da equipe } \\
\text { de enfermagem. }\end{array}$ & $\mathbf{n}$ & 101 & 16 & 27 & 144 \\
\cline { 2 - 6 } $\begin{array}{l}\text { 37. Os médicos deste hospital geralmente compreendem } \\
\text { e apreciam o que a equipe de enfermagem faz. }\end{array}$ & $\mathbf{n}$ & $\mathbf{( 7 0 , 1 )}$ & $(11,1)$ & $(18,8)$ & $(100)$ \\
\cline { 2 - 6 } & $\mathbf{( \% )}$ & $(46,5)$ & $(11,1)$ & $(42,4)$ & $(100)$ \\
\hline $\begin{array}{l}\text { 39. Os médicos desse hospital subestimam demais a } \\
\text { equipe de enfermagem. }\end{array}$ & $\mathbf{n}$ & 67 & 13 & 64 & 144 \\
\cline { 2 - 6 } & $\mathbf{( \% )}$ & $(46,5)$ & $(9)$ & $(44,4)$ & $(100)$ \\
\hline
\end{tabular}




\begin{tabular}{|l|c|c|c|c|c|}
\hline Interação com equipe de enfermagem & & Concordo & Neutro & Discordo & Total \\
\hline $\begin{array}{l}\text { 3. Em meu serviço, o pessoal da enfermagem se dispõe } \\
\text { e se ajudam uns aos outros "quando as coisas estão } \\
\text { corridas". }\end{array}$ & $\mathbf{n}$ & 131 & 2 & 11 & 144 \\
\cline { 4 - 7 } & $\mathbf{( \% )}$ & $(91)$ & $(1,4)$ & $(7,6)$ & $(100)$ \\
\hline $\begin{array}{l}\text { 10. Em minha unidade, é difícil para a(o)s enfermeiras(as) } \\
\text { novas(os) sentirem-se 'a vontade'. }\end{array}$ & $\mathbf{n}$ & 49 & 13 & 82 & 144 \\
\cline { 2 - 6 } & $\mathbf{( \% )}$ & $(34)$ & $(9)$ & $(56,9)$ & $(100)$ \\
\hline $\begin{array}{l}\text { 16. Em meu serviço, existe muito trabalho em equipe } \\
\text { e cooperação entre os vários níveis do pessoal de } \\
\text { enfermagem. }\end{array}$ & $\mathbf{n}$ & 109 & 4 & 31 & 144 \\
\hline $\begin{array}{l}\text { 23. Em meu serviço, o pessoal de enfermagem não é tão } \\
\text { amigável e extrovertido quanto eu gostaria. }\end{array}$ & $\mathbf{n}$ & $(75,7)$ & $(2,8)$ & $(21,5)$ & $(100)$ \\
\cline { 4 - 7 } & $\mathbf{( \% )}$ & $(24,3)$ & $(8,3)$ & $(67,4)$ & $(100)$ \\
\hline $\begin{array}{l}\text { 28. Em minha unidade há muita 'distinção de posições': } \\
\text { a(o)s enfermeira(o)s raramente 'misturam-se' com } \\
\text { aqueles de menor experiência ou diferentes tipos de } \\
\text { formação educacional. }\end{array}$ & $\mathbf{n}$ & 11 & 10 & 123 & 144 \\
\hline
\end{tabular}

Relacionado à interação com a equipe médica e de enfermagem, os enfermeiros possuem boa interação com a equipe de enfermagem e médica, existe cooperação e ajuda entre os profissionais, porém os enfermeiros gostariam que os médicos tivessem mais respeito e reconhecimento pelo trabalho exercido pela enfermagem. Destaca-se "q35: Eu gostaria que os médicos daqui mostrassem mais respeito pelas habilidades e conhecimentos da equipe de enfermagem" (70,1\% de concordância), "q16: Em meu serviço, existe muito trabalho em equipe e cooperação entre os vários níveis do pessoal de enfermagem" (75,7\% de concordância).

O Quadro 3 apresenta a distribuição das respostas dos componentes remuneração e status profissional, de acordo com o ISP.

Quadro 3 - Distribuição das respostas dos componentes Remuneração e Status Profissional. Santa Maria, RS, Brasil, 2015 (continua)

\begin{tabular}{|c|c|c|c|c|c|}
\hline REMUNERAÇÃO & & Concordo & Neutro & Discordo & Total \\
\hline \multirow[t]{2}{*}{ 1. Meu salário atual é satisfatório. } & $\mathbf{n}$ & 114 & 2 & 28 & 144 \\
\hline & $(\%)$ & $(79,2$ & $(1,4)$ & $(19,4)$ & $(100)$ \\
\hline \multirow{2}{*}{$\begin{array}{l}\text { 8. Tenho a impressão que grande parte do pessoal de } \\
\text { enfermagem desse hospital está insatisfeita com o seu } \\
\text { salário. }\end{array}$} & $\mathbf{n}$ & 48 & 25 & 71 & 144 \\
\hline & $(\%)$ & $(33,3$ & $(17,4)$ & $(49,3)$ & $(100)$ \\
\hline \multirow{2}{*}{$\begin{array}{l}\text { 14. Considerando o que é esperado do pessoal do serviço } \\
\text { de enfermagem, neste hospital o salário que recebemos } \\
\text { é razoável. }\end{array}$} & $\mathbf{n}$ & 87 & 8 & 49 & 144 \\
\hline & $(\%)$ & $(60,4$ & $(95,6)$ & (34) & $(100)$ \\
\hline \multirow{2}{*}{$\begin{array}{l}\text { 21. Neste hospital, o índice atual de reajuste salarial do } \\
\text { pessoal de enfermagem não é satisfatório. }\end{array}$} & $\mathbf{n}$ & 85 & 19 & 40 & 144 \\
\hline & $(\%)$ & (59 & $(13,2)$ & $(27,8)$ & $(100)$ \\
\hline \multirow{2}{*}{$\begin{array}{l}\text { 44. Neste hospital, se faz necessário um reajuste de } \\
\text { salários para o pessoal de enfermagem. }\end{array}$} & $\mathbf{n}$ & 100 & 20 & 24 & 144 \\
\hline & $(\%)$ & $(69,4$ & $(13,9)$ & $(16,7)$ & $(100)$ \\
\hline STATUS PROFISSIONAL & & Concordo & Neutro & Discordo & Total \\
\hline \multirow{2}{*}{$\begin{array}{l}\text { 9. A maioria das pessoas reconhece a importância da } \\
\text { assistência de enfermagem aos pacientes hospitalizados. }\end{array}$} & $n$ & 104 & 4 & 36 & 144 \\
\hline & $(\%)$ & $(72,2$ & $(2,8)$ & (25) & $(100)$ \\
\hline \multirow{2}{*}{$\begin{array}{l}\text { 11. Em minha mente, não tenho dúvidas: o que eu faço em } \\
\text { meu trabalho é realmente importante. }\end{array}$} & $n$ & 135 & 3 & 6 & 144 \\
\hline & $(\%)$ & $(93,8$ & $(2,1)$ & $(4,2)$ & $(100)$ \\
\hline \multirow{2}{*}{$\begin{array}{l}\text { 27. O que eu faço em meu trabalho realmente não } \\
\text { acrescenta nada significativo. }\end{array}$} & $n$ & 7 & 1 & 136 & 144 \\
\hline & (\%) & $(4,9$ & $(0,7)$ & $(94,4)$ & (100) \\
\hline \multirow{2}{*}{$\begin{array}{l}\text { 34. Eu me sinto orgulhosa(o) quando falo com outras } \\
\text { pessoas sobre o que eu faço no meu trabalho. }\end{array}$} & $\mathrm{n}$ & 129 & 9 & 6 & 144 \\
\hline & (\%) & $(89,6$ & $(6,3)$ & $(4,2)$ & (100) \\
\hline
\end{tabular}




\begin{tabular}{l|c|c|c|c|c|}
\hline 38. Se eu tivesse que decidir tudo outra vez, ainda assim & $\mathbf{n}$ & 106 & 14 & 24 & 144 \\
\cline { 2 - 6 } eu entraria na enfermagem. & $\mathbf{( \% )}$ & $(73,6$ & $(9,7)$ & $(16,7)$ & $(100)$ \\
\hline $\begin{array}{l}\text { 41. Meu trabalho, em particular, realmente não requer } \\
\text { muita habilidade ou conhecimento específico. }\end{array}$ & $\mathbf{n}$ & 4 & 1 & 139 & 144 \\
\cline { 2 - 7 } & $\mathbf{( \% )}$ & $(2,8$ & $(0,7)$ & $(96,5)$ & $(100)$ \\
\hline
\end{tabular}

Em relação ao componente remuneração, os profissionais expressaram que estão satisfeitos com seu salário atual, ainda mais se comparado ao salário recebido em outras instituições hospitalares. Entretanto gostariam de um reajuste salarial. Cita-se "q1: Meu salário atual é satisfatório" (79,2\% de concordância) e questão 44 "Neste hospital, se faz necessário um reajuste de salários para o pessoal de enfermagem" (69,4\% de concordância).

Quanto ao status profissional, os enfermeiros afirmam que o que fazem é importante e requer muito conhecimento e habilidades, porém gostariam de mais reconhecimento e valorização pelo trabalho que exercem. Ressalta-se "q11: Em minha mente, não tenho dúvidas: o que eu faço em meu trabalho é realmente importante" (93,8\% de concordância).

O Quadro 4 demonstra a distribuição das respostas dos componentes requisitos do trabalho e normas organizacionais, de acordo com o ISP.

Quadro 4 - Distribuição das respostas dos componentes Requisitos do Trabalho e Normas Organizacionais. Santa Maria, RS, Brasil, 2015 (continua)

\begin{tabular}{|c|c|c|c|c|c|}
\hline REQUISITOS DO TRABALHO & & Concordo & Neutro & Discordo & Total \\
\hline \multirow{2}{*}{$\begin{array}{l}\text { 4. Neste hospital, o pessoal de enfermagem tem muito } \\
\text { trabalho administrativo e burocrático. }\end{array}$} & $\mathbf{n}$ & 83 & 15 & 46 & 144 \\
\hline & $(\%)$ & $-57,6$ & $-10,4$ & $-31,9$ & -100 \\
\hline \multirow{2}{*}{$\begin{array}{l}\text { 15. Eu acho que poderia realizar um trabalho melhor se } \\
\text { não tivesse tanto o que fazer, o tempo todo. }\end{array}$} & $\mathbf{n}$ & 97 & 5 & 42 & 144 \\
\hline & $(\%)$ & $-67,4$ & $-3,5$ & $-29,2$ & -100 \\
\hline \multirow{2}{*}{$\begin{array}{l}\text { 22. Eu estou satisfeita(o) com os tipos de atividades que } \\
\text { realizo em meu trabalho. }\end{array}$} & $\mathbf{n}$ & 127 & 3 & 14 & 144 \\
\hline & $(\%)$ & $-88,2$ & $-2,1$ & $-9,7$ & -100 \\
\hline \multirow{2}{*}{$\begin{array}{l}\text { 24. Eu tenho tempo suficiente e oportunidades para } \\
\text { discutir os problemas da assistência ao paciente com } \\
\text { outros membros da equipe de enfermagem. }\end{array}$} & $\mathbf{n}$ & 65 & 7 & 72 & 144 \\
\hline & $(\%)$ & $-45,1$ & $-4,9$ & -50 & -100 \\
\hline \multirow{2}{*}{$\begin{array}{l}\text { 29. Eu tenho tempo suficiente para a assistência direta ao } \\
\text { paciente. }\end{array}$} & $\mathbf{n}$ & 57 & 12 & 75 & 144 \\
\hline & $(\%)$ & $-39,6$ & $-8,3$ & $-52,1$ & -100 \\
\hline \multirow{2}{*}{$\begin{array}{l}\text { 36. Eu poderia prestar uma assistência muito melhor se } \\
\text { tivesse mais tempo com cada paciente. }\end{array}$} & $\mathbf{n}$ & 116 & 7 & 21 & 144 \\
\hline & $(\%)$ & $-80,6$ & $-4,9$ & $-14,6$ & -100 \\
\hline NORMAS ORGANIZACIONAIS & & Concordo & Neutro & Discordo & Total \\
\hline \multirow{2}{*}{$\begin{array}{l}\text { 5. Em meu hospital, a equipe de enfermagem tem controle } \\
\text { suficiente sobre a programação de seu próprio turno de } \\
\text { trabalho. }\end{array}$} & $\mathbf{n}$ & 97 & 9 & 38 & 144 \\
\hline & $(\%)$ & $-67,4$ & $-6,3$ & $-26,4$ & -100 \\
\hline \multirow{2}{*}{$\begin{array}{l}\text { 12. Existe uma grande lacuna entre a administração } \\
\text { deste hospital e os problemas diários do serviço de } \\
\text { enfermagem. }\end{array}$} & $\mathbf{n}$ & 104 & 11 & 29 & 144 \\
\hline & $(\%)$ & $-72,2$ & $-7,6$ & $-20,1$ & -100 \\
\hline \multirow{2}{*}{$\begin{array}{l}\text { 18. Neste hospital não existem oportunidades suficientes } \\
\text { de promoção de pessoal de enfermagem. }\end{array}$} & $\mathbf{n}$ & 68 & 16 & 60 & 144 \\
\hline & $(\%)$ & $-47,2$ & $-11,1$ & $-41,7$ & -100 \\
\hline \multirow{2}{*}{$\begin{array}{l}\text { 25. Há grande oportunidade para a equipe de enfermagem } \\
\text { de participar do processo de tomada de decisões } \\
\text { administrativas. }\end{array}$} & $\mathbf{n}$ & 35 & 20 & 89 & 144 \\
\hline & $(\%)$ & $-24,3$ & $-13,9$ & $-61,8$ & -100 \\
\hline \multirow{2}{*}{$\begin{array}{l}\text { 33. Neste hospital, as decisões administrativas interferem } \\
\text { muito na assistência ao paciente. }\end{array}$} & $\mathbf{n}$ & 86 & 22 & 36 & 144 \\
\hline & $(\%)$ & $-59,7$ & $-15,3$ & -25 & -100 \\
\hline \multirow{2}{*}{$\begin{array}{l}\text { 40. Eu tenho todo o poder que quero no planejamento } \\
\text { de normas e procedimentos deste hospital e da minha } \\
\text { unidade. }\end{array}$} & $\mathbf{n}$ & 47 & 16 & 81 & 144 \\
\hline & $(\%)$ & $-32,6$ & $-11,1$ & $-56,3$ & -100 \\
\hline
\end{tabular}


42. A(s) chefia(s) de enfermagem geralmente consulta(m) a equipe nos problemas diários e procedimentos.

\begin{tabular}{|c|c|c|c|c|}
\hline $\mathbf{n}$ & 67 & 11 & 66 & 144 \\
\hline $\mathbf{( \% )}$ & $-46,5$ & $-7,6$ & $-45,8$ & -100 \\
\hline
\end{tabular}

Os Requisitos do trabalho são as atividades executadas rotineiramente pelos profissionais, os participantes estão satisfeitos com os tipos de atividades que realizam, contudo possuem muito trabalho administrativo e burocrático, e deixam as atividades assistenciais em segundo plano pela falta de tempo nos cuidados diretos aos pacientes. Demonstra-se "q36: Eu poderia prestar uma assistência muito melhor se tivesse mais tempo com cada paciente" (80,6\% de concordância).

Relacionado às normas organizacionais, os enfermeiros acreditam que a equipe de enfermagem tem controle sobre a programação de seu turno de trabalho e tomada de decisões dentro da unidade em que estão inseridos, mas se sentem limitados quanto às tomadas de decisões referentes à instituição hospitalar. Evidencia-se "q12: Existe uma grande lacuna entre a administração deste hospital e os problemas diários do serviço de enfermagem" (72,2\% de concordância).

\section{DISCUSSÃO}

$\mathrm{Na}$ análise da satisfação profissional, concebida pelo instrumento ISP, percebe-se a autonomia como um componente presente e satisfatório para os enfermeiros participantes. A autonomia equivale à independência profissional, a qual possibilita aos enfermeiros liberdade para tomada de decisões dentro de seus conhecimentos e também permite que possam realizar tarefas que tragam resultados satisfatórios ao seu trabalho. Alguns pontos favoráveis para a obtenção da autonomia são a constituição de saberes e conhecimentos, habilidades, cuidado holístico, educação em saúde, entre outros ${ }^{(12)}$.

A interação com as equipes de enfermagem e médica existe, os profissionais se relacionam bem e cooperam entre si, todavia as enfermeiras não se sentem respeitadas pelos médicos quanto a seus conhecimentos e habilidades. Este achado igualmente ocorreu junto a enfermeiros do Serviço de Atendimento Móvel de Urgência (SAMU) em Natal, os quais afirmam que gostariam que os médicos mostrassem mais respeito pelas habilidades e conhecimentos da equipe de enfermagem. Este fato pode ser resultado da superioridade histórica que os médicos exercem nas tomadas de decisão em relação aos enfermeiros ${ }^{(13)}$.

Em relação à interação da equipe de enfermagem, estudo mostra, igualmente a esta pesquisa, que há colaboração entre os vários níveis de pessoal da enfermagem e a equipe se ajuda quando as "coisas estão corridas no trabalho" ${ }^{\prime 13)}$.

A remuneração é um fator relevante para a satisfação profissional, os participantes consideraram seu salário atual satisfatório, mas gostariam que fosse reajustado. A baixa remuneração pode ser grande motivadora de insatisfação profissional ${ }^{(1)}$. Os baixos salários remetem a necessidade de mais de um vínculo empregatício, geralmente ocasionando sobrecarga que interfere na qualidade da assistência prestada e satisfação profissional(14).

Referente ao Status Profissional, quando os enfermeiros são reconhecidos evalorizados desenvolvem seus fazeres com maior empenho e satisfação. O reconhecimento e o prestígio social podem fazer com que os enfermeiros se sintam satisfeitos e plenos no desempenho de suas atividades laborais. A satisfação pode ser um estímulo para a realização da assistência com maior qualidade e a falta de valorização causa descontentamento e carência de motivação aos enfermeiros ${ }^{(15)}$.

Embora os enfermeiros tenham dito que estão satisfeitos com as atividades exercidas no trabalho, afirmam ter muito trabalho administrativo e burocrático. As condições de burocratização, inflexibilidade, rotinização e sobrecarga no trabalho são apontadas como causadoras de insatisfação nas equipes de enfermagem ${ }^{(1,9-10)}$. Os requisitos do trabalho possuem relação direta com as condições de trabalho, riscos ocupacionais, estresse, entre outros ${ }^{(16)}$.

Quanto às normas organizacionais, as limitações existentes entre os serviços e os servidores sobre assuntos administrativos e gerenciais podem interferir no trabalho e bem-estar da equipe de enfermagem. Para ocorrer a transformação de um trabalho que exige grande empenho em uma 
atividade prazerosa e qualificada, é preciso flexibilizar a organização e normas do trabalho, de modo que haja a adequação das normas organizacionais levando em consideração as necessidades dos trabalhadores, dando-Ihes maior liberdade para organizar seu serviço ${ }^{(17)}$.

\section{CONCLUSÃO}

Cabe ressaltar como limitações do estudo a demora na devolução dos instrumentos e a dificuldade na aceitação dos enfermeiros para participar do estudo. Por se tratar de um hospital escola,os participantes se encontravam saturados devido ao grande número de pesquisas realizadas no local. Vale lembrar que este estudo foi realizado em apenas uma instituição hospitalar, assim, a realidade retratada na pesquisa é singular, pois busca a percepção de vivências de cada sujeito.

É importante que o ambiente de trabalho, além de proporcionar remuneração, também propicie prazer para quem o exerce, levando em conta os aspectos organizacionais e subjetivos, garantindo satisfação aos profissionais para que eles se sintam motivados a realizar um trabalho com qualidade.

O estudo possibilitou a construção do conhecimento de enfermagem, assimilação de problemas presentes nos serviços de saúde, bem como para a reflexão dos membros da equipe de saúde sobre possível aprimoramento das relações no ambiente laboral, organização dos serviços e na qualidade dos atendimentos prestados.

Assim, as instituições hospitalares precisam se adequar melhor às necessidades dos profissionais, para que estes possam exercer seu trabalho com mais satisfação e qualidade. Os profissionais também devem se empenhar para que mudanças ocorram em seu ambiente de trabalho, cobrando e colaborando para melhorias da instituição.

\section{REFERÊNCIAS}

1. Paiva FFS, Rocha AM, Cardoso LDF. Satisfação profissional entre enfermeiros que atuam na assistência domiciliar. Rev. esc. enferm. USP. 2011; 45(6): 1452-8.

2. da Silva RM, Beck CLC, Guido LA, Lopes LFD, dos Santos JLG. Análise quantitativa da satisfação profissional dos enfermeiros que atuam no período noturno. Texto Contexto Enferm. 2009; 18(2): 298-305.

3. Oliveira ECL. Satisfação profissional, qualidade de serviço e segurança do utente: um estudo de caso em instituições de economia social[dissertação]. Coimbra: Universidade de Coimbra; 2015.

4. de Moura LLF. Qualidade de vida no trabalho: uma aplicação prática do modelo de Walton no contexto de uma empresa em Picos-PI [monografia].Picos (PI): Universidade Federal do Piauí; 2011.

5. Fraser TM. Human stress, work and job satisfaction: a critical approach. Geneva: International Labour Office; 1983.

6. Martinez MC, Paraguay AIBB. Satisfação e saúde no trabalho: aspectos conceituais e metodológicos. Cad. psicol. soc. trab. 2003; (6): 59-78.

7. Stamps PL. Using the index of work satisfaction in an organizational setting. Nurses and work satisfaction: an index for measurement. $2^{a}$ ed. Chicago: Health Administration Press; 1997.

8. Hill MM, Hill A. Investigação por questionário. Lisboa: Editora Sílado; 2002.

9. Lino MM. Satisfação profissional entre enfermeiras de UTI: adaptação transcultural do index of work satisfaction (IWS) [dissertação]. São Paulo (SP): Universidade de São Paulo; 1999.

10. Lino MM. Qualidade de vida e satisfação profissional de enfermeiras de unidades de terapia intensiva [tese]. São Paulo (SP): Universidade de São Paulo; 2004.

11. Ministério da Saúde (BR). Conselho Nacional de Saúde. Diretrizes e normas regulamentadoras de pesquisa 
envolvendo seres humanos. Resolução n. 466, de 12 de dezembro de 2012. Brasília; 2012.

12. Monteiro AI, dos Santos ADB, de Macedo IP, Gurgel PKF, Cavalcante JMP. A expressão da autonomia do enfermeiro no acompanhamento do crescimento e desenvolvimento da criança. Rev.enferm. UERJ. 2011; 19(3): 426-31.

13. Campos RM, de Farias GM, Ramos CS. Satisfação profissional da equipe de enfermagem do SAMU/Natal. Rev. Eletr. Enf. 2009; 11(3): 647-57.

14. Mauro MYC, da Paz AF, Mauro CCC, Pinheiro MAS, Silva VG. Condições de trabalho da enfermagem nas enfermarias de um hospital universitário. Esc. Anna Nery. 2010; 14(2): 13-8.

15. da Silveira RS, Funck CR, Lunardi VL, Avila LI, Lunardi Filho WD,Vidal DAS. Percepção dos trabalhadores de enfermagem acerca da satisfação no contexto do trabalho na UTI. EnfermFoco. 2012; 3(2): 93-6.

16. Siqueira VTA, Kurcgant P. Satisfação no trabalho: indicador de qualidade no gerenciamento de recursos humanos em enfermagem. Rev. esc. enferm. USP. 2012; 46(1): 151-7.

17. Kessler AI, Krug SBF. Do prazer ao sofrimento no trabalho da enfermagem: o discurso dos trabalhadores. Rev. Gaúcha Enferm. 2012; 33(1): 49-55. 